\title{
Subluxação atlantoaxial em 14 cães (2003-2008) ${ }^{1}$
}

\author{
Diego V. Beckmann ${ }^{2}$, Alexandre Mazzanti ${ }^{3^{*}}$, Giancarlo Santini², Rosmarini P. \\ Santos $^{2}$, Rafael Festugato ${ }^{2}$, Charles R. Pelizzari², Dakir Polidoro Neto ${ }^{4}$ e \\ Raquel Baumhardt ${ }^{4}$
}

\begin{abstract}
Beckmann D.V., Mazzanti A., Santini G., Santos R.P., Festugatto R., Pelizzari C., Polidoro Neto D. \& Baumhardt R. 2010. [Atlantoaxial subluxation in 14 dogs (2003-2008).] Subluxação atlantoaxial em 14 cães (2003-2008). Pesquisa Veterinária Brasileira 30(2):172-176. Departamento de Clínica de Pequenos Animais, Centro de Ciências Rurais, Universidade Federal de Santa Maria, Av. Roraima 1000, Camobi, Santa Maria, RS 97105-900, Brazil. E-mail: alexamazza@yahoo.com.br

A retrospective study on atlantoaxial subluxation in dogs was done by reviewing the cases filed from 2003 to 2008 in the neurological records of the Veterinary Hospital of the Universidade Federal de Santa Maria, at Santa Maria, Rio Grande do Sul, Brazil. The following data were identified: Breed, sex, age, etiology, clinical signs, duration of clinical course, assessment of the therapy employed and its efficacy, response to treatment and relapse. Fourteen dogs were diagnosed as affected by atlantoaxial subluxation and the condition was more frequent in dogs under twenty-four month old years and of toy breeds, such as Poodle (35.7\%), Pinscher (21.4\%) and Yorkshire terrier (21.4\%). The main cause found for the instability was agenesis of the odontoid process. Clinical signs ranged from cranial cervical pain to non-ambulatory tetraparesis. The predominant treatment employed was surgical which demonstrated to be efficacious in $90 \%$ of the cases with minor risks of relapse when compared with clinical treatment. The predominant time of recovery was 30-60 days after surgery. No correlation was found between the duration of clinical signs before surgery and the time of recovery.
\end{abstract}

INDEX TERMS: Atlantoaxial instability, neurology, disease of dogs.

RESUMO.- O objetivo deste trabalho foi realizar um estudo retrospectivo dos casos de subluxação atlantoaxial em cães, por meio de consulta dos registros neurológicos do Hospital Veterinário Universitário (HVU), entre os anos de 2003 e 2008. Foram identificados a raça, o sexo, a idade, a etiologia, os sinais neurológicos, a duração dos sinais clínicos, o tratamento empregado, a resposta ao tratamento, o tempo de recuperação, a recidiva e a relação entre a duração dos sinais clínicos e a recuperação pós-operatória. Foram feitos o diagnóstico de subluxação

\footnotetext{
${ }^{1}$ Recebido em 17 de julho de 2009.

Aceito para a publicação em 24 de janeiro de 2010.

2 Programa de Pós-Graduação em Medicina Veterinária, área de concentração em Cirurgia, CCR, UFSM, RS

${ }^{3}$ Departamento de Clínica de Pequenos Animais, Centro de Ciências Rurais (CCR), Universidade Federal de Santa Maria (UFSM), Av. Roraima 1000, Camobi, Santa Maria, RS 97105-900, Brasil. "Autor para correspondência: alexamazza@yahoo.com.br

${ }^{4}$ Curso de Medicina Veterinária, CCR, UFSM, Santa Maria, RS.
}

atlantoaxial em 14 cães, sendo as raças Poodle (35,7\%), Pinscher $(21,4 \%)$ e Yorkshire Terrier $(21,4 \%)$ as mais acometidas e a maioria $(92,8 \%)$ com idade inferior a 24 meses. A principal causa da instabilidade foi a agenesia do processo odontoide do áxis $(71,4 \%)$ e os sinais clínicos variaram desde hiperestesia cervical até tetraparesia não ambulatória. $O$ tratamento predominante foi o cirúrgico, que demonstrou ser eficaz com recuperação satisfatória em $90 \%$ dos casos e menor possibilidade de recidiva, quando comparado ao trata,mento clínico. O tempo de recuperação predominante foi de 30-60 dias após a cirurgia, não existindo relação deste com a duração dos sinais clínicos.

TERMOS DE INDEXAÇÃO: Instabilidade atlantoaxial, neurologia, doenças de cães.

\section{INTRODUÇÃO}

A subluxação atlantoaxial, também conhecida como instabilidade atlantoaxial, é uma afecção cuja causa princi- 
pal geralmente é congênita (Sturges 2009). Essa condição tem sido descrita em diferentes espécies, tais como ovinos, equinos, cervídeos, gatos e cães (Palmer 2002). Em cães, ocorre principalmente nos jovens de raças miniaturas (Beaver et al. 2000, Sturges 2009). De acordo com Sharp \& Wheeler (2005), vários processos patológicos podem conduzir à doença, tais como agenesia ou hipoplasia do processo odontoide (ou dente), fratura ou separação do processo odontoide e deficiência de ligamentos (ausência ou ruptura).

Os sinais clínicos variam desde dor cervical à tetraplegia e morte por insuficiência respiratória e decorrem de compressão aguda ou crônica da medula espinhal (Platt et al. 2004). Ocasionalmente, podem ser encontrados sinais clínicos associados à lesão no tronco encefálico caudal como hipoventilação e síndrome vestibular (Sturges 2009). O diagnóstico definitivo é dado pela avaliação dos sinais neurológicos, que permite localizar a lesão no segmento cervical da medula espinhal $\left(\mathrm{C}_{1}-\mathrm{C}_{5}\right)$, associada com técnicas de imagem da coluna vertebral cervical, principalmente radiografias nas posições látero-lateral e ventro-dorsal (Haid 2001, Havig et al. 2005, Sturges 2009). De acordo com Bynevelt et al. (2000), os exames de tomografia e ressonância magnética fornecem uma avaliação mais exata de alterações no processo odontoide do áxis.

O tratamento cirúrgico para a subluxação atlantoaxial está indicado para a permanente redução e estabilização da articulação com eliminação da compressão e prevenção do movimento vertebral (Shires 1998). O tratamento clínico inclui restrição de movimentos em gaiola, aplicação de colete cervical e uso de anti-inflamatórios (Havig et al. 2005).

Este estudo teve como objetivo avaliar retrospectivamente os casos de subluxação atlantoaxial em cães atendidos no Hospital Veterinário Universitário (HVU) da Universidade Federal de Santa Maria (UFSM), entre os anos de 2003 e 2008, na busca de identificar os aspectos epidemiológicos (raça, sexo e idade), etiológicos, clínicos (sinais neurológico e duração dos sinais clínicos), terapêuticos (tratamento empregado e resposta ao tratamento) e prognósticos (tempo de recuperação, recidiva e a relação entre a duração dos sinais clínicos e o tempo de recuperação pós-operatória).

\section{MATERIAL E MÉTODOS}

Foram revisados todos os protocolos de atendimento dos casos de subluxação atlantoaxial de cães ocorridos entre janeiro de 2003 e dezembro de 2008 e arquivados no Setor de Arquivo do HVU-UFSM. Foram incluídos apenas os casos diagnosticados pelo Serviço de Neurologia dessa instituição. A confirmação dos casos foi obtida pelo exame neurológico que definiu o local da lesão (C1-C5), pelo estudo radiográfico simples em posição látero-lateral e ventro-dorsal (Shires 1998, Haid 2001, Lorigados et al. 2004) e, em alguns casos, durante o procedimento cirúrgico. Informações gerais sobre raça, sexo, idade, etiologia, sinais neurológicos, duração dos sinais clínicos, tratamento empregado, resposta ao tratamento, tempo de recuperação e recidiva foram revisadas e anotadas.
O exame neurológico, compreendido pela avaliação do estado mental, da locomoção, das reações posturais, dos nervos cranianos e dos reflexos segmentares espinhais e pela palpação epaxial, foi realizado em todos os cães. Foi atribuída uma classificação em graus, antes dos tratamentos clínico e cirúrgico, de acordo com os sinais neurológicos, como segue: Grau V hiperestesia cervical com locomoção normal; Grau IV - ataxia; Grau III - tetraparesia ambulatória; Grau II - tetraparesia não ambulatória; e Grau I - tetraplegia (Platt et al. 2004). O exame radiográfico simples foi realizado em duas posições (ventrodorsal e látero-lateral) com o cão sob anestesia geral com cuidados para se evitar compressão medular durante a intubação. A posição ventro-dorsal foi empregada para avaliar alterações no processo odontoide. A posição látero-lateral foi utilizada para observar a angulação do processo odontoide e o posicionamento do processo espinhoso do áxis em relação ao arco dorsal do atlas.

Os tratamentos avaliados foram: clínico (uso de antiinflamatórios esteroidais ou não esteroidais [AINES], com ou sem restrição de movimentos em gaiola e com ou sem uso de colete cervical) ou cirúrgico (artrodese atlantoaxial mediante cirurgia de estabilização com uso de pinos de Steinmann e resina acrílica auto-polimerizável) (Shires 1998, Sharp \& Wheeler 2005).

Os proprietários dos cães foram contatados através de telefonemas para a obtenção do tempo de recuperação (caminhar sem quedas) após o procedimento cirúrgico e do retorno dos sinais clínicos (recidiva). A duração dos sinais clínicos antes da primeira consulta e o tempo de recuperação após o tratamento cirúrgico foi definido como: menor do que 30 dias; entre 30 e 60 dias e maior do que 60 dias.

O resultado do tratamento foi considerado satisfatório (cães com ausência de hiperestesia cervical e que voltaram a caminhar sem quedas), parcialmente satisfatório (cães com ausência de hiperestesia cervical e que voltaram a caminhar com auxílio) ou insatisfatório (cães sem melhora dos sinais clínicos neurológicos).

A duração dos sinais clínicos e o tempo de recuperação pós-operatório foram submetidos à análise estatística de correlação linear simples com nível de significância de $p<0,05$.

\section{RESULTADOS}

Composição da população estudada. Durante o período estudado (2003-2008), foram observados $14 \mathrm{ca}$ sos com diagnóstico de subluxação atlantoaxial, cujos resultados encontram-se no Quadro 1.

Idade, raça e sexo. A idade dos cães acometidos variou entre cinco e 60 meses e a média foi 16,9 meses $( \pm 14,2)$. Oito cães $(57,2 \%$ dos casos) apresentaram a doença com menos de 12 meses de idade, cinco $(35,7 \%)$ entre 12 e 24 meses e um $(7,1 \%)$ acima de 24 meses de idade. As raças acometidas foram: Poodle (5/14 [35,7\%]), Pinscher $(3 / 14[21,4 \%])$, Yorkshire Terrier $(3 / 14[21,4 \%])$ e Maltês (1/14 [7,1\%]). Dois cães (14,3\%) não tinham raça definida. A doença ocorreu em 10 fêmeas $(71,4 \%)$ e quatro machos $(28,6 \%)$.

Etiologia. As causas de subluxação atlantoaxial encontradas incluíram: agenesia do processo odontoide (10/ $14[71,5 \%])$, hipoplasia do processo odontoide (3/14 $[21,4 \%])$ e deslocamento dorsal do processo odontoide do áxis $(1 / 14[7,1 \%])$. 


\section{Quadro 1. Representação quanto à raça, sexo, idade, etiologia, sinais neurológicos, duração dos sinais clínicos, resposta ao tratamento e tempo de recuperação de 14 cães com subluxação atlantoaxial atendidos HVU-UFSM entre 2003 e 2008}

\begin{tabular}{lccccccc}
\hline $\begin{array}{l}\text { Raça/ano de } \\
\text { atendimento }\end{array}$ & Sexo & $\begin{array}{c}\text { Idade } \\
\text { (meses) }\end{array}$ & Etiologia & $\begin{array}{c}\text { Grau de } \\
\text { disfunção } \\
\text { neurológica }\end{array}$ & $\begin{array}{c}\text { Duração dos } \\
\text { sinais clínicos } \\
\text { (dias) }\end{array}$ & $\begin{array}{c}\text { Resposta ao } \\
\text { tratamento }\end{array}$ & $\begin{array}{c}\text { Tempo de } \\
\text { recuperação } \\
\text { (dias) }\end{array}$ \\
\hline 1. Poodle/2006 & F & 9 & Agenesia & III & 21 & Satisfatória & 43 \\
2. Poodle/2006 & F & 9 & Agenesia & II & 37 & Satisfatória & 52 \\
3. Poodle/2004 & F & 60 & Agenesia & II & 44 & Satisfatória & 42 \\
4. Poodle/2004 & F & 24 & Agenesia & III & 26 & Satisfatória & 33 \\
5. Poodle/2003 & F & 24 & Agenesia & III & 38 & Morte & - \\
6. Yorkshire/2003 & F & 9 & Agenesia & III & 18 & Satisfatória & 74 \\
7. Yorkshire/2005 & F & 21 & Agenesia & III & 34 & Satisfatória & 62 \\
8. Yorkshire/2006 & M & 7 & Agenesia & V & 66 & Satisfatória & 15 \\
9. Pinscher/2005 & F & 7 & Agenesia & III & 33 & Satisfatória & 39 \\
10. Pinscher/2005 & M & 20 & Hipoplasia & IV & 56 & Morte & - \\
11. SRD/2007 & F & 7 & Hipoplasia & II & 42 & Parcialmente satisfatória & 90 \\
12. SRD/2005 & M & 17 & DDDA & III & 53 & Satisfatória & 23 \\
13. Maltês/2008 & F & 5 & Agenesia & III & 24 & Satisfatória* & 21 \\
14. Pinscher/2008 & M & 11 & Hipoplasia & IV & 210 & * & $* *$ \\
\hline
\end{tabular}

M: macho; F: fêmea; SRD: sem raça definida; DDDA: Deslocamento dorsal do processo odontoide do áxis.

* Animal submetido ao tratamento clínico, conforme recomendações de Sharp \& Wheeler (2005).

** Cão sem tratamento por opção do proprietário.

Sinais neurológicos. Os sinais clínicos variaram de hiperestesia cervical à tetraparesia não ambulatória. Todos os cães apresentavam hiperestesia cervical pelo teste de movimentação do pescoço em flexão, extensão e lateralidade durante o fornecimento de alimentos. Quanto ao grau de disfunção neurológica, foi verificado: Grau $\mathrm{V}$ (1/14 [7,1\%]), Grau IV ([2/14] 14,3\%), Grau III (8/14 [57,1\%]) e Grau II (3/14 [21,5\%]).

Duração dos sinais clínicos. A duração dos sinais clínicos antes da primeira consulta foi: $<30$ dias em quatro cães $(28,6 \%)$, entre 30 e 60 dias em oito cães $(57,1 \%)$ e >60 dias, em dois cães (14,3\%), sendo a média de 48,9 dias $( \pm 49,3)$ e a variação de $21-210$ dias.

Tratamentos. Um cão (n-8) com apenas dor cervical (Grau V) foi submetido ao tratamento clínico composto por AINES, repouso em gaiola e colete cervical. Uma cadela (noำ) que apresentava tetraparesia ambulatória (Grau III) foi submetida ao mesmo tratamento clínico. Em um cão ( $n$ ำ14) que se encontrava em Grau IV não foi feito nenhum tratamento por opção do proprietário. Nos demais 11 cães submetidos ao tratamento cirúrgico de estabilização da articulação atlantoaxial, dois ( $n \div 5$ e 10) morreram durante a cirurgia e as necropsias revelaram agenesia e hipoplasia do processo odontoide, respectivamente.

Resposta aos tratamentos. Nos dois cães submetidos ao tratamento clínico, em um (nº8) a resposta foi considerada insatisfatória decorridos 30 dias, optando-se pela artrodese atlanto-axial. Na cadela (n-13), após 27 dias de terapia conservativa, a recuperação foi considerada satisfatória. Dos cães submetidos ao tratamento cirúrgico e com acompanhamento pós-operatório $(n=10)$, foi observado que nove $(90 \%)$ tiveram recuperação satisfatória e um ( $n$ ํ11) teve a recuperação considerada parcialmente satisfatória.

Tempo de recuperação e recidiva. $\mathrm{O}$ tempo de recu- peração no tratamento cirúrgico foi o seguinte: $<30$ dias em dois cães (20\%), entre 30 e 60 dias em cinco cães $(50 \%)$ e $>60$ dias em três cães (30\%), sendo a média de 47,3 dias $( \pm 22,9)$. O tempo de recuperação no único caso de tratamento clínico foi inferior a 30 dias. Não houve diferença significativa entre a duração dos sinais clínicos e o tempo de recuperação pós-operatória. Em nenhum dos cães avaliados foi observada recidiva dos sinais neurológicos.

\section{DISCUSSÃO}

Neste estudo, as raças Poodle, Pinscher e Yorkshire Terrier foram as mais acometidas por subluxação atlantoaxial e a maioria $(92,8 \%)$ com idade igual ou inferior a 24 meses. Estes resultados são semelhantes aos encontrados por outros autores (Beaver et al. 2000, Lorigados et al. 2004, Havig et al. 2005, Sturges 2009), que comentaram também a ocorrência da doença em raças de grande porte. Assim, deve-se salientar a importância da suspeita clínica de subluxação atlantoaxial em cães das raças de pequeno porte, com menos de dois anos de idade e que apresentam hiperestesia cervical.

A maioria dos cães deste estudo apresentou doenças congênitas como causa da subluxação atlantoaxial e a agenesia do processo odontoide foi mais frequente que a hipoplasia $(71,5 \%$ versus $21,4 \%)$ e o deslocamento dorsal ( $71,5 \%$ versus $7,1 \%)$. Foi descrito por Braund (1994), que a subluxação atlantoaxial pode ter algum fator hereditário envolvido em linhagens de cães de raças miniaturas na qual a anomalia congênita é comum, principalmente em cães jovens. Por outro lado, Beaver et al. (2000) salientaram que as causas traumáticas podem ocorrer em qualquer raça e idade.

Em apenas um caso, uma cadela com cinco anos de idade apresentando tetraparesia não ambulatória ( $n-3)$, foi registrado histórico de trauma e, ao observar a radiografia 
ventro-dorsal, pode-se constatar a agenesia do processo odontoide. Em teoria, por se tratar de etiologia congênita, os sinais clínicos deveriam ser manifestos ainda quando filhotes ( $<12$ meses). Assim, este achado, por nós considerado atípico, serve de alerta aos clínicos para a ocorrência da doença também em cães adultos. Para alguns autores (Sturges 2009), a maioria dos casos de subluxação atlantoaxial ocorre em cães acima de um ano. A maneira de viver da paciente, sob restrição de movimento, revelado no histórico clínico, pode justificar a não ocorrência desta doença precocemente. Para Lorenz \& Kornegay (2006), alguns cães podem não demonstrar sinais clínicos devido ao suporte vertebral adequado de outras estruturas fibrosas e musculares, que evitam a subluxação, sendo que, o menor trauma pode resultar em subluxação do processo odontoide do áxis, compressão da medula espinhal e alteração neurológica (Havig et al. 2005).

A subluxação atlantoaxial foi evidente em exame radiográfico nas incidências látero-lateral (com o cão em posição neutra) e em posição ventro-dorsal (com o tubo endotraqueal removido), para uma avaliação completa do processo odontoide do áxis (Bynevelt et al. 2000). O diagnóstico de deslocamento dorsal do processo odontoide foi dado através de radiografia simples látero-lateral e confirmado durante o procedimento cirúrgico. Este achado provavelmente ocorreu pela ausência ou ruptura do ligamento transverso do áxis.

De acordo com Shires (1998), cães de ambos os sexos podem ser afetados pela subluxação atlantoaxial, entretanto, conforme outro estudo, realizado por Lorigados et al. (2004), fêmeas são mais acometidas. Os casos aqui descritos afetaram predominantemente fêmeas; mas pelo pequeno número de cães aqui descritos, não se pode concluir estatisticamente quanto à predisposição sexual.

A variação dos sinais clínicos observada neste estudo se deve aos diferentes níveis de compressão da medula espinhal, sendo a hiperestesia cervical, a ataxia proprioceptiva (inicialmente mais evidente nos membros pélvicos) e a deficiência proprioceptiva as mais encontradas em casos brandos de compressão medular. Já a tetraplegia raramente é verificada, pois, segundo Sharp \& Wheeler (2005), uma lesão na medula espinhal desta gravidade geralmente conduz à deficiência respiratória e morte. Em um cão (n¹4) que se encontrava em Grau IV, o proprietário notou locomoção diferente, caracterizada por ataxia proprioceptiva nos quatro membros desde os sete meses de idade. Neste caso, o diagnóstico da doença foi determinado durante uma consulta clínica por outro motivo. Deve-se salientar a importância do exame da locomoção em pacientes com suspeita de doenças neurológicos a fim de auxiliar na determinação da localização da lesão, conforme salientado por Parent (2004)

Para Stainki et al. (1998), a hiperestesia cervical é intensificada pela flexão do pescoço quando o cão é forçado ao movimento. Sharp \& Wheeler (2005), no entanto, recomendaram evitar este tipo de manipulação durante 0 exame neurológico para não agravar o quadro clínico. A maneira empregada para avaliar a hiperestesia nos cães deste estudo foi através do oferecimento de alimentos palatáveis em diferentes posições (lateral, dorsal e ventral) e concomitante observação da limitação do pescoço durante a tentativa de apreensão, conforme indicado por Da Costa et al. (2006).

Embora o tratamento clínico seja recomendado nos casos de subluxação atlantoaxial, no atual estudo, em um cão (n-1) foi realizada este tipo de terapia composta por anti-inflamatório esteroidal, repouso em gaiola e o uso de colete cervical, conforme recomendações de Havig et al. (2005). Decorridos 30 dias, no entanto, o paciente foi reavaliado e o resultado foi considerado insatisfatório, optando-se pelo tratamento cirúrgico. Por outro lado, em uma cadela $\left(\mathrm{n}^{\circ} 13\right)$ foi realizada terapia semelhante por apresentar idade inferior a seis meses (Sturges 2009) e o resultado foi considerado satisfatório após 27 dias. Provavelmente, esta diferença na resposta entre os cães esteja no diagnóstico precoce. Já os demais pacientes, no entanto, o histórico revelou que todos tinham sido submetidos à terapia clínica somente com a administração de antiinflamatório esteroidal ou não esteroidal sem a complementação com a restrição de movimentos e contenção do pescoço, ao contrário do recomendado por Sharp \& Wheeler (2005). Diante disso, neste estudo, a eficiência do tratamento clínico não pode ser avaliada, pois a maioria dos pacientes não estava de acordo com a recomendada pela literatura.

O tratamento cirúrgico compreendido por colocação de dois pinos de Steimann mais resina acrílica autopolimerizável demonstrou ser eficaz, sendo indicado para a maioria dos pacientes com subluxação atlantoaxial que apresentam deficiências neurológicas moderadas a graves (Sharp \& Wheeler 2005).

O óbito durante a cirurgia em dois cães foi em decorrência de complicações durante a anestesia. O posicionamento do paciente e o acesso cirúrgico ventral próximo ao tronco vago-simpático, que pode ser estimulado pela distração da musculatura do pescoço pelo afastador auto-estático, provavelmente contribuíram para esta ocorrência. Segundo Cybulsky \& D'Angelo (1988), é imperativa a monitoração destes pacientes devido às variações nas frequências cardíaca e de pressão arterial que ocorrem durante cirurgia na coluna cervical. Apesar de não haver diferença significativa entre a duração dos sinais clínicos e o tempo de recuperação pós-operatória, salienta-se a importância do diagnóstico precoce á fim de se evitar disfunções neurológicas graves e respostas insatisfatórias ao tratamento.

\section{CONCLUSÕES}

Por meio dos estudos feitos nos registros médicos do HVU-UFSM, pode-se concluir em relação à subluxação atlantoaxial que:

- Cadelas miniaturas, como Poodle, Pinscher e Yorkshire Terrier, e com idade inferior a 24 meses são mais afetadas; 
- A causa mais frequente da subluxação atlantoaxial é a agenesia do processo odontoide do áxis;

- O principal sinal neurológico encontrado é a hiperestesia cervical;

- A duração dos sinais neurológicos varia entre 21 e 210 dias;

- O tratamento predominante cirúrgico demonstra ser eficaz, com menor possibilidade de recidiva;

- Não há relação entre a duração dos sinais clínicos e o tempo de recuperação pós-operatória.

\section{REFERÊNCIAS}

Beaver D.P., Ellison G.W., Lewis D.D., Goring R.L., Kubilis P.S. \& Barchard C. 2000. Risk factors affecting the outcome of surgery for atlantoaxial subluxation in dogs: 46 cases (1978-1998). J. Am. Vet. Med. Assoc. 216(7):1104-1109.

Braund K.G. 1994. Neurological diseases, p.81-332. In: Ibid. (Ed.), Clinical Syndromes in Veterinary Neurology. Mosby, St Louis.

Bynevelt M., Rusbridge C. \& Britton J. 2000. Dorsal dens angulation and a chiari type malformation in a Cavalier King Charles Spaniel. Vet. Radiol. Ultrasound 41(6):521-524.

Cybulsky G. \& D’Angelo C.M. 1988. Neurological deterioration after laminectomy for spondylotic cervical myeloradiculopathy: The putative role of spinal cord ischaemia. J. Neurol. Psych. 51:717-718.

Da Costa R.C., Parent J., Dobson H., Holmberg D. \& Partlow G. 2006. Comparison of magnetic resonance imaging and myelography in 18 Doberman Pinscher dogs with cervical spondylomyelopathy. Vet. Radiol. Ultrasound 47(6):523-531.
Haid R.W. 2001. C1-C2 transarticular screw fixation: Technical aspects. Neurosurg. 49(1):71-74.

Havig M.E., Cornell K.K., Hawthorne J.C., McDonnell J.J. \& Selcer B.A. 2005. Evaluation of nonsurgical treatment of atlantoaxial subluxation in dogs: 19 cases (1992-2001). J. Am. Vet. Med. Assoc. 227(2):257-262.

Lorenz M.D. \& Kornegay J.N. 2006. Tetraparesia, hemiparesia e ataxia, p.175-218. In: Ibid. (Ed.), Neurologia Veterinária. 4ª ed. Manole, São Paulo.

Lorigados C.A.B., Sterman F.A. \& Pinto A.C.B.F. 2004. Estudo clínico radiográfico da subluxação atlantoaxial congênita em cães. Braz. J. Vet. Res. Anim. Sci. 41(6):368-374.

Palmer M.V. 2002. Atlantoaxial instability in a white-tailed deer fawn (Odocoileus virginianus). J. Wildl. Dis. 38(4):860-862.

Parent J. 2004. Ataxia e paresia, p.147-149. In: Ettinger S.J. \& Feldman E.C. (Eds), Tratado de Medicina Interna. 5 ${ }^{\mathrm{a}}$ ed. Guanabara Koogan, Rio de Janeiro.

Platt S.R., Chambers J.N. \& Cross A. 2004. A modified ventral fixation for surgical management of atlantoaxial subluxation in 19 dogs. Vet. Surg. 33(4):349-354.

Sharp N.J.H. \& Wheeler S.J. 2005. Small Animal Spinal Disorders: Diagnosis and surgery. $2^{\text {nd }}$ ed. Elsevier Mosby, Philadelphia.

Shires P.K. 1998. Instabilidade atlantoaxial, p.1261-1272. In: Slatter (Ed.), Manual de Cirurgia de Pequenos Animais. $2^{\mathrm{a}}$ ed. Manole, São Paulo.

Stainki D.R., Garcia F.S. \& Silva N.R. 1998. Instabilidade atlantoaxial em canino: breve revisão e relato de caso. Revta Fac. Zootec. Vet. Agron., Uruguaiana, 5(1):77-81.

Sturges B.K. 2009. Diagnosis and treatment of atlantoaxial subluxation, p.1083-1087. In: Bonagura J.D. \& Twedt D.C. (Eds), Kirk's Current Veterinary Therapy. $14^{\text {th }}$ ed. Saunders, St Louis. 